\title{
LETTERS
}

\section{Case did not consider all potential causes of chilblains}

I read the CMAJ Practice article on severe acute respiratory syndrome coronavirus 2 (SARS-CoV-2)-related chilblains in a 16-year-old female by Ladha and Dupuis ${ }^{1}$ with interest, but it raised a few concerns. In females in this age group, the association between anorexia nervosa and pernio or chilblains has been noted for several decades. ${ }^{2,3}$

This case report makes no mention of the patient's body habitus or body mass index, and there is no description of a physical examination, which is seminal in ruling out autoimmune disorders, such as lupus, or malignant disease. The laboratory investigations are thorough, but a normal chest radiograph and computed tomography scan of the abdomen would have added weight to the final diagnosis of SARS-CoV-2-related chilblains. The dermal pathology of pernio or chilblains is nonspecific ${ }^{4}$ and, therefore, a skin biopsy is not suggested. ${ }^{4}$

There is no description of the patient's smoking history, including e-cigarette usage ("vaping"), as nicotine will exacerbate the vasospasm seen in pernio or chilblains. $^{4}$

During the current coronavirus disease 2019 pandemic, clinicians are adopting a "hands-off" or telehealth approach to reviewing patients, including history taking. However, there are individuals for whom an office visit and physical examination is important. This may be one of those cases: the positive result for SARSCoV-2 antibody serology testing may be incidental and unrelated to her skin condition. $^{5}$

\section{Catharine L. Dewar PhD MD}

Rheumatologist (retired), Lions Gate

Hospital, Vancouver, BC

- Cite as: CMAJ 2020 September 8;192:

E1046. doi: $10.1503 / \mathrm{cmaj} .76376$

\section{References}

1. Ladha MA, Dupuis EC. SARS-CoV-2-related chilblains. CMAJ 2020;192:E804.

2. Rustin MHA, Foreman JC, Dowd PM. Anorexia nervosa associated with acromegaloid features, onset of acrocyanosis and Raynaud's phenomenon and worsening of chilblains. $J$ R Soc Med 1990;83:495-6.

3. White KP, Rothe MJ, Milanese A, et al. Perniosis in association with anorexia nervosa. Pediatr Dermatol 1994;11:1-5.

4. Whitman PA, Crane JS. Pernio (chilblains). In: StatPearls [Internet]. Treasure Island (FL): StatPearls Publishing; 2020. Available: www.ncbi. nlm.nih.gov/books/NBK549842/ (accessed 2020 July 2).

5. Ladha MA, Dupuis EC. The authors respond to: "Case did not consider all potential causes of chilbains" [letter]. CMAJ 2020;192:E1047.

Competing interests: None declared. 